\title{
MATRICES OVER ORTHOMODULAR LATTICES
}

\author{
by J. H. BEVIS
}

(Received 27 December, 1967)

In this paper elementary properties are established for matrices whose coordinates are elements of a lattice $L$. In particular, many of the results of Luce [4] are extended to the case where $L$ is an orthomodular lattice, a lattice with an orthocomplementation denoted by ' in which $a \leqq b \Rightarrow a \vee\left(a^{\prime} \wedge b\right)=b$. Originally, these were called orthocomplemented weakly modular lattices, Foulis [2]. In Theorem 1 a characterization is given of the nucleus with respect to matrix multiplication, which is in general nonassociative. Matrices with $A^{-1}=\operatorname{transpose}(A)$ are characterized in Lemma 8 . Theorems 3 and 4 respectively, give partial characterizations of zero divisors and inverses.

1. $\mathscr{A}_{m n}(L)$ and $\mathscr{A}_{n}(L)$ will denote, respectively, the set of all $m \times n$ matrices and the set of all $n \times n$ matrices whose coordinates are elements of $L$. The argument $L$ will be suppressed when it is not needed. Except for $L$, capital letters denote matrices. $A_{i j}$ will denote the $(i, j)$ th element of the matrix $A$. For matrices of suitable size $A \vee B, A \wedge B$, and, if $L$ has a complementation, $A^{\prime}$ are defined coordinatewise. $A \leqq B \Leftrightarrow A_{i j} \leqq B_{i j}$ for all $i, j$. For conformal matrices define the product by $(A B)_{i j}=\mathrm{V}_{k}\left(A_{i k} \wedge B_{k j}\right)$. We assume that $L$ has a least element $o$ and a greatest element 1. Define $0, I$, and $E$ to be the matrices with $0_{i j}=o$, $I_{i j}=1, E_{i j}=o$ for $i \neq j$, and $E_{i i}=1$, the sizes of these and other matrices being determined by the context, if not otherwise restricted. $\mathscr{A}_{m n}(L)$ is lattice isomorphic to the direct product of $L$ with itself $m n$ times; hence, if $L$ is orthomodular, then so is $\mathscr{A}_{m n}(L)$. The proof of the first lemma is elementary and is therefore omitted.

LEMMA 1. For matrices of appropriate size,

(i) $B A \vee C A \leqq(B \vee C) A, A B \vee A C \leqq A(B \vee C), A(B \wedge C) \leqq A B \wedge A C,(B \wedge C) A \leqq B A \wedge C A$,

(ii) $B \leqq C \Rightarrow A B \leqq A C$ and $B A \leqq C A$,

(iii) $0 \wedge A=0 A=A 0=0, E A=A E=A, A \leqq A I \leqq I, A \leqq I A \leqq I$.

In an orthocomplemented lattice we say that $a$ commutes with $b$ and write $a \mathscr{C} b$ if $\left(a \vee b^{\prime}\right) \wedge b=a \wedge b$. The centre of $L$ is defined as $\mathscr{C}(L)=\{a \in L \mid a \mathscr{C} b$ for all $b \in L\}$. Many of the results of Foulis [3] concerning the relation $\mathscr{C}$ will be used without making specific references. In particular, great use will be made of the Foulis-Holland theorem which states that, if $L$ is an orthomodular lattice and two of the three relations $a \mathscr{C} b, a \mathscr{C} c, b \mathscr{C} c$ hold, then $(a \vee b) \wedge c=(a \wedge c) \vee(b \wedge c)$ and $(a \wedge b) \vee c=(a \vee c) \wedge(b \vee c)$. If $L$ is orthocomplemented, then so is $\mathscr{A}_{m n}(L)$, and in this case we have $A \mathscr{C} B \Leftrightarrow A_{i j} \mathscr{C} B_{i j}$ for all $i, j$ and

$$
\mathscr{C}\left(\mathscr{A}_{m n}\right)=\left\{A \in \mathscr{A}_{m n} \mid A_{i j} \in \mathscr{C}(L)\right\}
$$

These concepts should not be confused with matrix multiplication commuting or the multiplicative centre of $\mathscr{A}_{n}$. For the remainder of this section we assume that $L$ is orthomodular. 
LemMA 2. Given $A$ in $\mathscr{A}_{m n}, A \in \mathscr{C}\left(\mathscr{A}_{m n}\right)$ if and only if any one (and thus all) of the following hold for all $B, C$ in $\mathscr{A}_{p q}$, where $p$ or $q$ is chosen to make the multiplication conformal:

$$
B A \vee C A=(B \vee C) A, B C \vee A C=(B \vee A) C, A B \vee A C=A(B \vee C), B A \vee B C=B(A \vee C) .
$$

Proof. If $A \in \mathscr{C}\left(\mathscr{A}_{m n}\right)$ one may easily check the identities. Conversely, choose $B$ to have $o$ entries except for $B_{i l}=b$ and $C$ to have $o$ entries except for $C_{i i}=b^{\prime}$. Then $B A \vee C A=(B \vee C) A$ implies that $\left(b \wedge A_{i j}\right) \vee\left(b^{\prime} \wedge A_{i j}\right)=A_{i j}$. Thus $A_{i j} \in \mathscr{C}(L)$ for all $i$ and $j$. The other cases are similar.

CoRollary. Matrix multiplication is distributive over joins in $\mathscr{A}_{n}(L)$ if and only if $L$ is Boolean.

Proof. This follows from the lemma, since $\mathscr{C}(L)=L \Leftrightarrow L$ is Boolean.

A collection $S=\left\{s_{\alpha} \mid \alpha \in \mathscr{I}\right.$, an indexing set $\}$ with each $s_{\alpha}$ in $L$, is said to have property $\mathscr{D}$ on $\mathscr{M}$, if whenever $x \in \mathscr{M}, x \wedge V_{\alpha} x_{\alpha}=V_{\alpha}\left(x \wedge x_{\alpha}\right)$, where $x_{\alpha} \in L\left(o, s_{\alpha}\right)=\left\{s \in L \mid s \leqq s_{\alpha}\right\}$. Note that, if $s_{\alpha}=o$ for all but possibly one $\alpha$ or if $L\left(o, s_{\alpha}\right) \subset \mathscr{C}(L)$ for all $\alpha$ and either $\mathscr{I}$ is finite or $L$ is complete, then $S$ has property $\mathscr{D}$ on $L$. If $S$ has property $\mathscr{D}$ on $L$ then $L\left(o, s_{\alpha} \wedge s_{\beta}\right)$ is distributive for each pair $\alpha, \beta$ of distinct indices. We say that a matrix $A$ in $\mathscr{A}_{m n}$ is right $\wedge$-distributive if $(B \wedge C) A=B A \wedge C A$ for all $B, C \in \mathscr{A}_{p m}$. For conformal matrices $(A, B, C)$ is an associative triple if $A(B C)=(A B) C$. By elementary calculations, $(A, B, C)$ is an associative triple if the entries in two of the three matrices are in $\mathscr{C}(L)$.

T. S. Blyth [1] has characterized $\wedge$-distributive matrices over a Boolean lattice. Lemma 3 and its corollary are generalizations of one of his results.

Lemma 3. Given $A$ in $\mathscr{A}_{m n}(L)$, for each $j=1, \ldots, n$, define

$$
\mathscr{M}_{j}=\left\{V_{i} x_{i} \mid x_{i} \in L\left(o, A_{i j}\right), i=1, \ldots, n\right\} \text {. }
$$

$A$ is right $\wedge$-distributive if and only if $A_{i j} \wedge A_{k j}=0$ for all $i, j, k$ with $i \neq k$, and for each $j$ the jth column of $A$ satisfies $\mathscr{D}$ on $\mathscr{M}_{j}$.

Proof. For sufficiency,

$$
\begin{aligned}
(B A \wedge C A)_{i j} & =\left[\mathrm{V}_{k}\left(B_{i k} \wedge A_{k j}\right)\right] \wedge\left[\mathrm{V}_{h}\left(C_{i h} \wedge A_{h j}\right)\right] \\
& =\mathrm{V}_{k}\left[B_{i k} \wedge A_{k j} \wedge \mathrm{V}_{h}\left(C_{i h} \wedge A_{h j}\right)\right] \\
& =\mathrm{V}_{k} \mathrm{~V}_{h}\left(B_{i k} \wedge A_{k j} \wedge C_{i h} \wedge A_{h j}\right)=\mathrm{V}_{k}\left(B_{i k} \wedge C_{i k} \wedge A_{k j}\right)=[(B \wedge C) A]_{i j}
\end{aligned}
$$

Conversely, set $B=E$ and $C=E^{\prime}$; then $0=\left(E \wedge E^{\prime}\right) A=A \wedge E^{\prime} A$ implies that $A_{i j} \wedge A_{k j}=o$ for all $i, j, k$ with $i \neq k$. If $B$ is chosen so that the join over $k$ has only one term, we obtain $(2)=(3)$ which implies that, for each $j$, the $j$ th column of $A$ satisfies $\mathscr{D}$ on $\bigcup_{i} L\left(0, A_{i j}\right)$. With this we obtain $(1)=(2)$ for any $B$ in $\mathscr{A}_{p m}$ and the necessity follows.

CoRollary. If $B \leqq A$ and $A$ is right $\wedge$-distributive, then $B$ is right $\wedge$-distributive.

Similar results are obtained for left $\wedge$-distributive matrices. For the rest of Section 1 , take $n \geqq 2$. 
Lemma 4. Let $A, B, C \in \mathscr{A}_{n}$.

(i) $(A, B, C)$ is an associative triple for all $B$ and $C$ if and only if $A \in \mathscr{C}\left(\mathscr{A}_{n}\right)$ and each row of A satisfies $\mathscr{D}$ on $L$.

(ii) $(B, C, A)$ is an associative triple for all $B$ and $C$ if and only if $A \in \mathscr{C}\left(\mathscr{A}_{n}\right)$ and each column of $A$ satisfies $\mathscr{D}$ on $L$.

(iii) $(B, A, C)$ is an associative triple for all $B$ and $C$ if and only if each row and column of $A$ satisfies $\mathscr{D}$ on $L$.

Proof. For necessity in (i), let $B$ have 1's in the $j$ th row and $o$ 's elsewhere. By examining the $(i, j)$ th element of the equation $(A B) C=A(B C)$, we obtain $V_{h}\left(C_{h k} \wedge A_{i j}\right)=A_{i j} \wedge V_{h} C_{h k}$. Thus $C$ may be chosen to imply that $A_{i j} \in \mathscr{C}(L)$ for any $i, j$. Now, with $A$ in $\mathscr{C}\left(\mathscr{A}_{n}\right)$, choose $C$ to have $o$ 's except in the $h$ th row, to obtain $\mathrm{V}_{k}\left(A_{i k} \wedge B_{k h} \wedge C_{h j}\right)=C_{h j} \wedge V_{k}\left(A_{i k} \wedge B_{k h}\right)$. This holds for all $B_{k h}$ and $C_{h j}$ if and only if the $i$ th row of $A$ satisfies $\mathscr{D}$ on $L$. For sufficiency, take the join with respect to $h$ of both sides of the above equation. The other parts of the lemma are obtained in a similar manner.

The nucleus of $\mathscr{A}_{n}$ is $\left\{A \in \mathscr{A}_{n} \mid\right.$ any triple containing $A$ is associative $\}$. If one defines a scalar meet by $(x \wedge A)_{i j}=x \wedge A_{i j}$ for $x$ in $L$, it may be shown by standard methods that $A B=B A$ for all $B$ in $\mathscr{A}_{n}$ if and only if there is an $a$ in $L$ such that $A=a \wedge E$. The multiplicative centre of $\mathscr{A}_{n}$ is defined as the set of all $A$ in the nucleus of $\mathscr{A}_{n}$ such that $A B=B A$ for all $B \in \mathscr{A}_{n}$. Since each row or column of $a \wedge E$ has only one nonzero element, the rows and columns of $a \wedge E$ satisfy $\mathscr{D}$ on $L$. We have obtained

THEOREM 1. The nucleus of $\mathscr{A}_{n}$ is $\left\{A \in \mathscr{C}\left(\mathscr{A}_{n}\right) \mid\right.$ rows and columns of $A$ satisfy $\mathscr{D}$ on $\left.L\right\}$. The multiplicative centre of $\mathscr{A}_{n}$ is $\{a \wedge E \mid a \in \mathscr{C}(L)\}$.

Corollary. Matrix multiplication is associative in $\mathscr{A}_{n}(L)$ if and only if $L$ is Boolean.

2. The transpose of a matrix $A$ is defined to be $A^{2}$ where $\left(A^{t}\right)_{i j}=A_{j i}$. It follows that $(A \vee B)^{t}=A^{t} \vee B^{t},(A \wedge B)^{t}=A^{t} \wedge B^{t}, A^{t t}=A,(A B)^{t}=B^{t} A^{t}$, for $A$ and $B$ of suitable size, and, if $L$ has a complementation, $\left(A^{\prime}\right)^{\prime}=\left(A^{\prime}\right)^{t}$. If $L$ is orthocomplemented, for square matrices we say that $A$ is symmetric if $A^{\prime} \perp A^{\prime}\left(A \perp B \Leftrightarrow A \leqq B^{\prime}\right.$; in this case $A$ and $B$ are said to be orthogonal), and $A$ is skew-symmetric if $A \perp A^{t}$.

Lemma 5. If $A \in \mathscr{A}_{n}(L)$ with $L$ orthocomplemented, then $A^{\prime} \perp A^{t} \Leftrightarrow A=A^{t}$.

Proof. By taking the transpose of $A^{t} \leqq A$, we obtain $A \leqq A^{t}$. Hence

$$
A^{\prime} \perp A^{t} \Leftrightarrow A^{t} \leqq A \Leftrightarrow A=A^{t} .
$$

Theorem 2. For $A$ in $\mathscr{A}_{n}(L)$ with $L$ orthomodular, $A$ has an orthogonal decomposition into a symmetric and skew-symmetric matrix (i.e., $A=S \vee Q$ with $S$ symmetric, $Q$ skew-symmetric, and $S_{\perp} Q$ ) if and only if $A^{\mathscr{C}} A^{\mathrm{t}}$. If the decomposition exists, it is unique.

Proof. Suppose that $S$ and $Q$ exist such that $A=S \vee Q, S_{\perp} Q, S_{\perp}^{\prime} S^{t}$ and $Q \perp Q^{\prime}$. Thus $A \wedge A^{t}=(S \vee Q) \wedge\left(S \vee Q^{t}\right)=S \vee\left(Q \wedge Q^{t}\right)=S$ and

$$
A \wedge A^{\prime \prime}=(S \vee Q) \wedge\left(S^{\prime} \wedge Q^{\prime \prime}\right)=Q \wedge S^{\prime} \wedge Q^{\prime \prime}=Q
$$

Hence, if the decomposition exists, it is unique. Now $A=\left(A \wedge A^{t}\right) \vee\left(A \wedge A^{z \prime}\right)$ implies that 
$A \mathscr{C} A^{t}$. If $A \mathscr{C} A^{t}$, then $A=\left(A \wedge A^{t}\right) \vee\left(A \wedge A^{t \prime}\right), A \wedge A^{t} \leqq A^{\prime} \vee A^{t}=\left(A \wedge A^{\prime \prime}\right)^{\prime},\left(A \wedge A^{t}\right)^{t}=A^{t} \wedge A$ and $A \wedge A^{t \prime} \leqq A \vee A^{t \prime}=\left(A^{t \prime} \wedge A\right)^{t \prime}$. Thus $S=A \wedge A^{t}$, and $Q=A \wedge A^{\prime \prime}$ is the required decomposition.

For $A, B$ in $\mathscr{A}_{n}(L)$ with $L$ orthocomplemented the following results are easily obtained. If $A$ and $B$ are skew-symmetric, then $A \wedge B$ is skew-symmetric. For $A$ and $B$ symmetric we obtain (i) $A \vee B, A \wedge B, A^{t}$ and $A^{\prime}$ are symmetric, (ii) $A B$ is symmetric if and only if $A B=B A$.

3. $A$ is called row (column) consistent if $A I=I(I A=I)$, where the $I$ 's may not have the same size. The proofs of the lemmas of this section are elementary and are therefore omitted.

Lemмa 6. The following are equivalent whenever $A$ is of appropriate size:

(i) $A$ is row (column) consistent.

(ii) $\mathrm{V}_{k} A_{i k}=1$ for all $i\left(\mathrm{~V}_{k} A_{k j}=1\right.$ for all $\left.j\right)$.

(iii) $E \leqq A A^{t}\left(E \leqq A^{t} A\right)$.

LEMMA 7. If $A B$ is row (column) consistent, then $A$ is row consistent ( $B$ is column consistent).

Corollary. If $A$ has a left (right) inverse, then $A$ is column (row) consistent and its left (right) inverse is row (column) consistent.

Let $L$ be the orthocomplemented modular lattice of subspaces of Euclidean 2-space. Let $A, B \in \mathscr{A}_{n}(L)$ be such that $A_{i j}, B_{i j} \neq o, 1$. If all of the elements of $A$ and $B$ are distinct, then $A$ and $B$ are both row and column consistent, but $A B=0$. Thus the converse of Lemma 7 that Luce [4] proved for $L$ Boolean, does not obtain in general. For $L$ Boolean, Rutherford [5] has shown that, for square matrices, $A$ has a one sided inverse $\Leftrightarrow A$ has a two sided inverse $\Leftrightarrow A^{-1}=A^{t}$; examples similar to the one above show that, if $A \notin \mathscr{C}\left(\mathscr{A}_{n}\right)$, then $A$ may have several one or two sided inverses. However the next lemma, which is due to Luce [4], holds for arbitrary lattices with $o$ and 1 .

Lemma 8. $A A^{t}=E\left(A^{t} A=E\right)$ if and only if $A$ is row (column) consistent and $A_{i \mathrm{k}} \wedge A_{j k}=0$ $\left(A_{k j} \wedge A_{k i}=o\right)$ for all $i, j, k$ with $i \neq j$.

4. In this section we assume that $L$ is orthomodular and that $B \in \mathscr{C}\left(\mathscr{A}_{m n}\right)$. Conditions are given for finding a matrix $X$ satisfying $X A \leqq B$ or $X A \geqq B$. The results can then be applied to the matrix equation $X A=B$. Dual statements are given for results concerning $A X \leqq B$, $A X \geqq B$, and $A X=B$.

Lemma 9. (i) If $X \wedge\left(B^{\prime} A^{t}\right)=0$, then $X A \leqq B$. If $\left(X, A, B^{\prime t}\right)$ is an associative triple, then $X A \leqq B \Rightarrow X \wedge\left(B^{\prime} A^{t}\right)=0$. (Note that $A$ in $\mathscr{A}_{p n}$ implies that $B^{\prime} A^{t}$ and $X$ are in $\mathscr{A}_{m p}$.) (ii) If $X \wedge\left(A^{t} B^{\prime}\right)=0$, then $A X \leqq B$. If $\left(B^{\prime \prime}, A, X\right)$ is an associative triple, then $A X \leqq B \Rightarrow X \wedge\left(A^{t} B^{\prime}\right)=0$. (Note that $A$ in $\mathscr{A}_{m p}$ implies that $A^{t} B^{\prime}$ and $X$ are in $\mathscr{A}_{p n}$ )

Proof. By examining the $(i, i)$ th element of the matrix product, one notes that

$$
X Y \leqq E^{\prime} \Leftrightarrow X \wedge Y^{t}=0 \Leftrightarrow Y X \leqq E^{\prime}, \text { for } X, Y^{t} \text { in } \mathscr{A}_{m p} .
$$

Now

$$
X \wedge\left(B^{\prime} A^{t}\right)=0 \Rightarrow X\left(A B^{\prime \prime}\right) \leqq E^{\prime} \Rightarrow X_{i k} \wedge A_{k j} \wedge B_{i j}^{\prime}=0 \text { for all } i, j, k
$$


Since $B \in \mathscr{C}\left(\mathscr{A}_{m n}\right)$, we obtain $X_{i k} \wedge A_{k j} \leqq B_{i j}$ and thus $(X A)_{i j} \leqq B_{i j}$. Conversely, $X A \leqq B$ implies that $o=X_{i k} \wedge A_{k j} \wedge B_{i j}^{\prime}=V_{k}\left(X_{i k} \wedge A_{k j} \wedge B_{i j}^{\prime}\right)=(X A)_{i j} \wedge\left(B^{t}\right)_{j i}^{\prime}$, the last step being accomplished since $B_{i j} \in \mathscr{C}(L)$. Taking the join over $j$, we obtain $E^{\prime} \geqq(X A) B^{\prime \prime}=X\left(A B^{\prime \prime}\right)$. The result follows from the remark at the beginning of the proof.

THEOREM 3. If $A$ is not row (column) consistent, then $A$ is a right (left) divisor of zero. If $A \in \mathscr{C}\left(\mathscr{A}_{p n}\right)\left(A \in \mathscr{C}\left(\mathscr{A}_{m p}\right)\right)$ or if $A \in \mathscr{A}_{p 1}\left(A \in \mathscr{A}_{1 p}\right)$, then $A$ is a right (left) divisor of zero if and only if $A$ is not row (column) consistent.

Proof. Set $B=0$ in $\mathscr{A}_{m n}$ and $B^{\prime t}=I$ in $\mathscr{A}_{n m}$. If $A$ is not row consistent, then $A I<I$ in $\mathscr{A}_{p m}$. Thus $0<X \leqq(A I)^{t \prime} \Rightarrow X \wedge(A I)^{t}=0 \Rightarrow X A=0 \Rightarrow A$ is a right zero divisor. Now $\mathrm{V}_{h} \mathrm{~V}_{k}\left(X_{i k} \wedge A_{k h}\right)=\bar{V}_{k}\left(X_{i k} \wedge V_{h} A_{k h}\right)$ and $(X, A, I)$ is an associative triple, if $n=1$ or if $A \in \mathscr{C}\left(\mathscr{A}_{\mathrm{pn}}\right)$ Hence in either case $X A=0$ implies that $X \wedge(A I)^{t}=0$. But, if $A$ is row consistent, $(A I)^{t}=I$ in $\mathscr{A}_{m p}$ and $X=X \wedge I=0$.

LEMmA 10. If there is a matrix $C$ such that $C \leqq A$ and $B \leqq I C(B \leqq C I)$, then any $X$ such that $X \geqq B C^{\prime}\left(X \geqq C^{\prime} B\right)$ is a solution of $X A \geqq B(A X \geqq B)$.

Proof. If $A, C \in \mathscr{A}_{p n}$, then $X, I \in \mathscr{A}_{m p}$. With $j, k=1, \ldots, n$,

$$
\left[\left(B C^{t}\right) A\right]_{i j}=\mathrm{V}_{h}\left[A_{h j} \wedge \mathrm{V}_{k}\left(B_{i k} \wedge C_{h k}\right)\right] \geqq \mathrm{V}_{h}\left(A_{h j} \wedge B_{i j} \wedge C_{h j}\right)=B_{i j} \wedge(I C)_{i j}=B_{i j}
$$

COROLlaRY. If $X \geqq I C^{t}\left(X \geqq C^{t} I\right)$, where $C \leqq A$ and $C$ is column (row) consistent, then $X A=I(A X=I)$.

Proof. Set $B=I$ in Lemma 10.

Lemma 11. $X A \geqq E(A X \geqq E)$ has a solution if and only if $A$ is column (row) consistent.

Proof. The result follows from Lemmas 6 and 7.

THEOREM 4. For square matrices, if $C^{t} \leqq X \leqq\left(E^{\prime} A^{t}\right)^{\prime}\left(C^{t} \leqq X \leqq\left(A^{t} E^{\prime}\right)^{\prime}\right)$, where $C \leqq A$ and $C$ is column (row) consistent, then $X$ is a left (right) inverse of $A$.

Proof. The result is obtained by letting $B=E$ in Lemmas 9 and 10 .

\section{REFERENCES}

1. T. S. Blyth, $\Lambda$-distributive Boolean matrices, Proc. Glasgow Math. Assoc. 7 (1965), 93-100.

2. D. J. Foulis, Baer *-semigroups, Proc. Amer. Math. Soc. 11 (1960), 648-654.

3. D. J. Foulis, A note on orthomodular lattices, Portugal. Math. 21 (1962), 65-72.

4. R. D. Luce, A note on Boolean matrix theory, Proc. Amer. Math. Soc. 3 (1952), 382-388.

5. D. E. Rutherford, Inverses of Boolean matrices, Proc. Glasgow Math. Assoc. 6 (1963), 49-53.

Virginia Polytechnic InStitute

Blacksburg, Virginia 